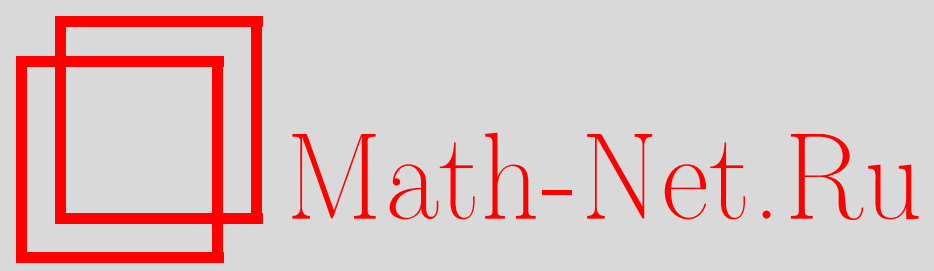

В. С. Владимиров, Математические вопросы теории нелинейных псевдодифференциальных уравнений $p$-адических струн, Вестн. Сам. гос. техн. ун-та. Сер. Физ.-мат. науки, 2011, выпуск 1(), 34-41

DOI: https://doi.org/10.14498/vsgtu929

Использование Общероссийского математического портала Math-Net.Ru подразумевает, что вы прочитали и согласны с пользовательским соглашением http: //www. mathnet.ru/rus/agreement

Параметры загрузки:

IP : 35.173 .219 .12

26 апреля 2023 г., $15: 16: 01$ 
УДК 517.968.4

\section{МАТЕМАТИЧЕСКИЕ ВОПРОСЫ ТЕОРИИ НЕЛИНЕЙНЫХ ПСЕВДОДИФФЕРЕНЦИАЛЬНЫХ УРАВНЕНИЙ р-АДИЧЕСКИХ СТРУН}

\section{В. С. Владимиров}

Математический институт им. В. А. Стеклова РАН, 119991, Москва, ул. Губкина, 8.

E-mail: vladim@mi.ras.ru

Работа посвящена математическому описанию динамики тахионов открытой, замкнутой и открыто-замкнутой р-адических струн. Обсуждаются вопросы существования и несуществования непрерывных решений и их свойства, а также структура нулей у решений. Получены новые многомерные нелинейные уравнения ультрапараболического типа. Приведён ряд нерешённых задач.

Ключевые слова: p-адические струны, нелинейные псевдодифберенциальные уравнения, существование и непрерывность решений, структура нулей у решенuй.

Введение. Для описания динамики тахионов открытой, замкнутой и открыто-замкнутой $p$-адических струн была предложена следующая упрощённая система нелинейных псевдодифференциальных уравнений движения [1-3]:

$$
\begin{gathered}
\psi^{p^{2}}=e^{-\square / 4} \psi, \\
\varphi^{p} \psi^{p(p-1) / 2}=e^{-\square / 2} \varphi,
\end{gathered}
$$

где $\psi(t, x)$ и $\varphi(t, x), x=\left(x_{1}, x_{2}, \ldots, x_{d-1}\right)$ - тахионные поля для открытых и замкнутых струн; $\square=-\partial_{t}^{2}+\nabla_{x}^{2}-d$-мерный Д'аламбертиан; $p-$ простое число (показатель нелинейности), $p=2,3,5, \ldots$ (Впредь $p$ и $p^{2}$ иногда будем считать целыми числами $n>1$.)

Система уравнений (1) разделилась на уравнение (1a), описывающее динамику $\psi$ замкнутой струны, и на уравнение $(1 \mathrm{~b})$, описывающее динамику $\varphi$ открытой струны при известном поле $\psi$.

При $\psi=1$ система (1) превращается в уравнение для открытой струны

$$
\varphi^{p}=e^{-\square / 2} \varphi
$$

При $d=1$ уравнения (1) принимают вид:

$$
\begin{gathered}
\psi^{p^{2}}=e^{\partial^{2} / 4} \psi, \\
\varphi^{p} \psi^{p(p-1) / 2}=e^{\partial^{2} / 2} \varphi, \\
\varphi^{p}=e^{\partial^{2} / 2} \varphi .
\end{gathered}
$$

В классе (измеримых) функций $(\psi, \varphi)$, удовлетворяющих условию

$$
|f(t)|=\left(e^{\varepsilon t^{2}}\right), \quad|t| \rightarrow \infty, \quad \varepsilon<1 / 2,
$$

Василий Сергеевич Владимиров (д.ф.-м.н., академик), главный научный сотрудник (советник РАН), отд. математической физики. 
уравнения (2) превращаются в классическую систему нелинейных интегральных уравнений

$$
\begin{gathered}
\psi^{p^{2}}(t)=\left(K_{1 / 4} \psi\right)(t), \quad t \in \mathbb{R}, \\
\varphi^{p}(t) \psi^{p(p-1) / 2}(t)=\left(K_{1 / 2} \varphi\right)(t), \quad t \in \mathbb{R}, \\
\varphi^{p}(t)=\left(K_{1 / 2} \varphi\right)(t), \quad t \in \mathbb{R} .
\end{gathered}
$$

Здесь

$$
\left(K_{\sigma} f\right)(t)=\frac{1}{\sqrt{4 \pi \sigma}} \int_{-\infty}^{\infty} \exp \left[-\frac{(t-\tau)^{2}}{4 \sigma}\right] f(\tau) d \tau, \quad t \in \mathbb{R}, \sigma>0
$$

есть полугруппа интегральных операторов теплопроводности, $K_{\sigma} K_{s}=K_{\sigma+s}$, $\sigma>0, s>0$.

Для уравнения (1a) ставятся следующие краевые условия:

$$
\lim _{t \rightarrow-\infty} \psi(t, x)=\lim _{t \rightarrow \infty} \psi(t, x)=1,
$$

а для уравнений $(1 \mathrm{~b})$ и $(1 \mathrm{c})-$

$$
\lim _{t \rightarrow-\infty} \varphi(t, x)=1, \quad \lim _{t \rightarrow \infty} \varphi(t, x)=\left\{\begin{aligned}
-1, & p \text { - нечётное; } \\
0, & p \text {-чётное }
\end{aligned}\right.
$$

и рассматриваются соответствующие краевые задачи.

Поставленные краевые задачи естественно изучать в некоторых алгебрах (обобщённых) функций $[4,5]$. Физический интерес представляют только вещественные непрерывные решения.

Исследованию этого нового класса уравнений с бесконечным числом производных посвящены многие работы с использованием компьютерной техники (см. [1-24] и литературу там). В струнной теории поля взаимодействие нелокально [6], что существенно отличает её от классической локальной теории поля. Они представляют значительный интерес не только для $p$-адической математической физики, но и для космологии [9-13].

1. Открытая струна, $\boldsymbol{d}=\mathbf{1}$. Динамика открытой струны определяется интегральным уравнением (3c) и граничными условиями (4b). Приведём некоторые утверждения о решениях $\varphi$ краевой задачи (3c), (4b) при нечётном $p$ [5]; при чётном $p$ законченных результатов известно мало [21].

Существование Решения [5]. Краевая задача (3c), (4b) не имеет знакопостоянных решений. При нечётном р она имеет нечётное непрерывное решение $\varphi(t)$ с простым нулем у функиии $\varphi^{p}(t)$.

СвЯЗь С УРАВНЕНИЕМ ТЕПЛОПРОВОДНОСТИ [21]. Краевая задача (3c), (4b) эквивалентна краевой задаче для уравнения теплопроводности

$$
\begin{aligned}
U_{\sigma}^{\prime} & =\frac{1}{2} U_{t t}^{\prime \prime}, \quad 0<\sigma \leqslant 1, \quad t \in \mathbb{R}, \\
U(0, t) & =\varphi(t), \quad U(1, t)=\varphi^{p}(t), \quad t \in \mathbb{R} .
\end{aligned}
$$

(Обратим внимание, что переменные $\sigma$ и $t$ в уравнении (5а) играют роль переменных $t$ и $x$ при сравнении с классическим уравнением теплопроводности.) 
Функция $U(\sigma, t)$ называется интерполирующей между решением $\varphi$ и его степенью $\varphi^{p}$. Она представляется формулой Пуассона для уравнения теплопроводности (5a):

$$
U(\sigma, t)=\frac{1}{\sqrt{2 \pi \sigma}} \int_{-\infty}^{\infty} \varphi(\tau) \exp \left[-\frac{(t-\tau)^{2}}{2 \sigma}\right] d \tau, \quad t \in \mathbb{R}, \quad 0<\sigma \leqslant 1 .
$$

Для нулей интерполирующей функции $U(\sigma, t)$ имеет место следующая теорема.

О ВетвЛЕНИИ нУЛЕЙ [21]. Пусть функиия $U(1, t)=\varphi^{p}(t)$ имеет в точке $t=0$ нуль чётной кратности $2 m$. Тогда уравнение

$$
U(1-\varepsilon, t)=0 \quad \text { nрu } \varepsilon \rightarrow+0
$$

имеет ровно $2 m$ различных простых вещественных нулей:

$$
t_{k}^{ \pm}(\varepsilon)= \pm \lambda_{k} \sqrt{\varepsilon}+O(\varepsilon), \quad k=1,2, \ldots, m,
$$

где $\lambda_{k}$ - положительные (простые) корни полинома Эрмита $H_{2 m}(\lambda)$.

Решения краевых задач допускают разложения по модифииированным полиномам Эрмита

$$
V_{n}(t)=2^{-\frac{n}{2}} H_{n}\left(\frac{t}{\sqrt{2}}\right), \quad n=0,1, \ldots
$$

Обозначим через $L_{2}^{\alpha}, \quad 0<\alpha$ гильбертово пространство измеримых квадратично-интегрируемых с весом

$$
d \mu_{\alpha}(t)=\sqrt{\frac{\alpha}{\pi}} e^{-\alpha t^{2}} d t
$$

функций на $\mathbb{R}$, со скалярным произведением

$$
(f, g)_{2}^{\alpha}=\int_{-\infty}^{\infty} f(t) \bar{g}(t) d \mu_{\alpha}(t)
$$

и нормой $\|f\|=\sqrt{(f, f)_{2}^{\alpha}}$.

РАЗЛОЖЕНИЯ ПО МОДИФИЦИРОВАННЫМ ПОЛИНОМАМ ЭрмИТА [21]. Если решение $\varphi$ уравнения (3c) принадлежит $L_{2}^{\alpha}$, то оно разлагается в ряд по полиномам Эрмита $V_{n}$ :

$$
\varphi(t)=\sum_{n=0}^{\infty} a_{n} 2^{n / 2} V_{n}(t), \quad a_{n}=\left(\varphi, V_{n}\right)_{2}^{1 / 2}
$$

а функиия $\varphi^{p}(t)-$ в рлд Тейлора:

$$
\varphi^{p}(t)=\sum_{n=0}^{\infty} a_{n} \frac{t^{n}}{n !},
$$


сходящийся локально-равномерно на $\mathbb{R}$.

Соотношения ортогонАЛьности. Пусть $\varphi(t)-$ решение уравнения (3c) $u t=0$ - нуль функции $\varphi^{p}(t)$ кратности s. Тогда справедливы следующие соотношения ортогональности [5]:

$$
\int_{-\infty}^{\infty} \varphi(\tau) \tau^{k} e^{-\frac{\tau^{2}}{2}} d \tau=\left\{\begin{aligned}
0, & k=0,1, \ldots, s-1 \\
\neq 0, & k=s
\end{aligned}\right.
$$

НЕЛОКАЛЬНЫЙ ЗАКОН СОХРАНЕНИЯ [21]. Пусть $p$ - нечётное $u U(\sigma, t)-$ интерполирующая функиия между решением $\varphi$ и его степенью $\varphi^{p}$. Тогда справедлив закон сохранения

$$
\int_{-\infty}^{\infty}[\varphi(t)-U(\sigma, t)] d t=0=\int_{-\infty}^{\infty}\left[\varphi(t)-\varphi^{p}(t)\right] d t, \quad \sigma>0 .
$$

При этом справедливъ включения

$$
1-|\varphi(\cdot)|, 1-|U(\sigma, \cdot)| \in L_{1}(\mathbb{R}), \quad \sigma \geqslant 0 .
$$

Важную роль играют нули функции $\varphi^{p}[23,24]$.

Нули функции $\varphi^{p}$. При нечётном $p$ число нулей функции $\varphi^{p}(t)$ задачи $(3 \mathrm{a}),(4 \mathrm{~b})$ конечно: $t_{1}, t_{2}, \ldots, t_{m} ;$ их кратности $s_{1}, s_{2}, \ldots, s_{m}$ конечны; число перемен знака решения $\varphi(t)$ не больше числа $m$ нулей функиии $\varphi^{p}(t)$.

При чётном р число нулей $m$ может быть бесконечным, а их кратности - удвоенные нечётные числа.

Доказательство утверждения о структуре и кратности нулей у решений сразу следует из утверждения «о ветвлении нулей» и из уравнения теплопроводности (5a).

Ответ на вопрос о гладкости решений даёт следующее утверждение.

ДИФФЕРЕНЦИАЛЬНЫЕ СВОЙСТВА РЕШЕНИЙ [21]. Если $p-$ неётно и $\varphi-p e$ шение краевой задачи (3c), (4b), то

$$
\varphi^{\prime} \in L_{1}(\mathbb{R}),
$$

причём вторая производная $\varphi^{\prime \prime}$ принадлежит $L_{2}(\mathbb{R})$ тогда и только тогда, когда

$$
s_{k}>p / 2, \quad k=1,2, \ldots, m .
$$

2. Замкнутая струна, $\boldsymbol{d}=1$. Динамика замкнутой струны определяется интегральным уравнением (3a) (с заменой $p$ на $p^{2}$ ) и граничными условиями (4a). Поэтому почти все утверждения п. 1 для открытой струны с естественными изменениями остаются справедливыми и для замкнутой струны [22], кроме включений (7) закона сохранения (6):

$$
\begin{gathered}
1-\psi(\cdot), 1-U(\sigma, \cdot) \in L_{1}(\mathbb{R}), \quad \sigma>0 ; \\
\int_{-\infty}^{\infty}[1-U(\sigma, t)] d t=\int_{-\infty}^{\infty}[1-\psi(t)] d t, \quad \sigma>0 .
\end{gathered}
$$


Как и в п. 1, имеет место аналогичная теорема о разложении решений по полиномам Эрмита (см. [21]).

РАЗлОЖЕНИЯ ПО ПОЛИНОМАм Эрмита. Если решение $\psi$ уравнения (3а) принадлежит $L_{2}^{1}$, то оно разлагается в ряд по полиномам Эрмита:

$$
\psi(t)=\sum_{n=0}^{\infty}\left(\psi, H_{n}\right)_{2}^{1} \frac{H_{n}(t)}{2^{n} n !}
$$

а функция $\psi^{p^{2}}(t)-$ в ряд Тейлора:

$$
\psi^{p^{2}}(t)=\sum_{n=0}^{\infty}\left(\psi, H_{n}\right) \frac{t^{n}}{n !},
$$

сходлщийся локально-равномерно на $\mathbb{R}$.

Отметим также, что число нулей у решений для замкнутой струны конечно, а их кратности - удвоенные нечётные числа при чётном $n$, а при нечётном $n$-как нечётные, так и удвоенные нечётные числа.

Для краевой задачи (3a), (4a) при чётном $p^{2}=4$ (или более общо с заменой $p^{2}$ на $n$ ) справедлив такой отрицательный результат, сформулированный в [14] и [24]: не существует непрерывных чётных неубывающих при $t>0$ решений этой задачи.

Открытым остаётся следующий вопрос: возможно ли существование непрерывных колеблющихся или разрывных (кусочно-непрерывных) решений этой задачи?

3. Открыто-замкнутая струна, $\boldsymbol{d}=\mathbf{1}$. Динамика открыто-замкнутой струны определяется системой уравнений $(3 \mathrm{a}),(3 \mathrm{~b})$ и граничными условиями (4). Пусть $\psi_{0}(t)$ - известное чётное решение краевой задачи (3a), (4a) такое, что кратности $s_{k}$ нулей функции $\psi_{0}^{p^{2}}(t)$ удовлетворяют неравенству

$$
s_{k}<\frac{2 p^{2}}{p-1}, \quad k=1,2, \ldots, m .
$$

Подставляя это решение в уравнение (3b), получим уравнение

$$
\varphi^{p}(t) \psi_{0}^{p(p-1) / 2}(t)=\left(K_{1 / 2} \varphi\right)(t)
$$

и граничные условия (4b). Применяя метод последовательных приближений к краевой задаче $(9),(4 \mathrm{~b})$, получим решение $\varphi_{0}$. В результате справедлива следующая теорема [3, 24].

О СУЩЕСТвОВАНИи РЕШЕния. Пусть $p=4 n+1$, где $n \geqslant 1-$ целое число, $\psi_{0}$-чётное решение краевой задачи (3a), (4a), причём кратности $s_{k}$, нулей функиии $\psi_{0}^{p^{2}}(t)$ удовлетворяют условию (8). Тогда непрерывное решение $\left(\psi_{0}, \varphi_{0}\right)$ краевой задачи (3a), (3b), (4) для открыто-замкнутой струны существует.

4. Многомерный случай, $\boldsymbol{d} \geqslant 2$. Рассмотрим уравнение

$$
\varphi^{n}(t, x)=\left(e^{-\sigma_{0} \square} \varphi\right)(t, x), \quad(t, x) \in \mathbb{R}^{d},
$$


объединяющее оба уравнения (1a) и (1c).

Решением уравнения (10) является функция

$$
\varphi(t, x)=p^{\frac{d-1}{2(p-1)}} \psi(t) \exp \left(-\frac{p-1}{p}|x|^{2}\right),
$$

где $\psi(t)$ - любое решение уравнений (3c).

Уравнение (11) рассматриваем на классе (обобщённых) функций $\varphi(t, x)$ таких, что их преобразование Фурье $\tilde{\varphi}(t, x)$ по $x$ удовлетворяет оценке

$$
|\tilde{\varphi}(t, x)| \leqslant e^{(1-\varepsilon) t^{2}}(1+|x|)^{1-d-\varepsilon}
$$

при любом $\varepsilon>0$. В этом случае псевдодифференциальное уравнение (10) эквивалентно интегральному уравнению

$$
\pi^{-(d-1) / 2} \int_{\mathbb{R}^{d-1}} e^{-|y|^{2}} \varphi^{p}(t, x-y) d y=\frac{1}{\sqrt{\pi}} \int_{-\infty}^{\infty} e^{-\tau^{2}} \varphi(t-\tau, x) d \tau .
$$

Таким образом, справедливо следующее утверждение [24].

ОБ ЭКВИВАЛЕНТНОСТИ УРАВНЕНИЙ. При условии (12) уравнение (10) эквивалентно интегральному уравнению (13).

СледствиЕ. Для сферически-симметричных $d \geqslant 3$ по $x$ решений $\varphi(t, r)$, $r=|x|$ уравнение (12) принимает следующий вид:

$$
\begin{gathered}
2 \int_{0}^{\infty} e^{-r^{2}-\rho^{2} \rho^{d-2}(r \rho)^{-(d-3) / 2} I_{(d-3) / 2}(2 r \rho) \varphi^{p}(t, \rho) d \rho=} \\
=\frac{1}{\sqrt{\pi}} \int_{-\infty}^{\infty} e^{-(t-\tau)^{2}} \varphi(\tau, r) d \tau, \quad d \geqslant 3 ; \\
2 \int_{0}^{\infty} e^{-r^{2}-\rho^{2}} \operatorname{ch}(2 r \rho) \varphi^{p}(t, \rho) d \rho= \\
\sqrt{\pi} \int_{-\infty}^{\infty} e^{-(t-\tau)^{2}} \varphi(\tau, r) d \tau, \quad d=2
\end{gathered}
$$

где $I_{\nu}(z)=\exp (-\pi \nu / i) J_{v}(i z)-\oint у н к u и я$ Бесселя мнимого аргумента.

ЗАмечаниЕ. Уравнение (13) удобно для численных расчётов с применением квадратурных формул Гаусса [24].

Краевые задачи для уравнения (10) эквивалентны краевым задачам для уравнения параболического типа с двумя временами, о чём говорится в следующем утверждении [24].

ОБ интеРпОлиРующЕй ФУнкции. Краевые задачи для уравнения (10) эквивалентны нелинейной краевой задаче для уравнения параболического типа с двумя временами $\sigma$ и $t$ для интерполирующей функици $U(\sigma, t, x)$ :

$$
\frac{\partial U}{\partial \sigma}=\frac{1}{4} \square U, \quad 0<\sigma<1, \quad(t, x) \in \mathbb{R}^{d}
$$

при начальных условиях

$$
U(0, t, x)=\varphi(t, x), \quad U(1, t, x)=\varphi^{p}(t, x), \quad(t, x) \in \mathbb{R}^{d}
$$


и граничных условиях (4a) или (4b) соответственно.

В работе [23] доказано несуществование почтипериодических по $t$ решений рассматриваемых краевых задач.

Автор благодарит проф. В. П. Радченко за редакторскую работу с рукописью статьи.

Работа выполнена при финансовой поддержке РФФИ (грант 08-01-00727) и гранта президента РФ (НШ-7675.2010.1).

\section{БИБЛИОГРАФИЧЕСКИЙ СПИСОК}

1. Brekke L., Freund P. G. O. p-Adic Numbers in Physics // Phys. Rep. (Rev. Set. Phys. Lett.), 1993. Vol. 233, no. 1. Pp. 1-66.

2. Moeller N., Schnabl M. Tachyon condensation in open-closed p-adic string theory // J. High Energy Phys., 2004. no. 1, 011. 18 pp.

3. Владимиров В.С. О нелинейных уравнениях p-адических открытых, замкнутых и открыто-замкнутых струн// TMФ, 2006. Т. 149, № 3. С. 354-367; англ. пер.: Vladimirov V.S. Nonlinear equations for p-adic open, closed, and open-closed strings // Theoret. and Math. Phys., 2006. Vol. 149, no. 3. Pp. 1604-1616.

4. Гельфанд И. М., Шилов Г.Е. Обобщённые функции. Т.2: Пространства основных и обобщённых функций. М.: Физматлит, 1958. 307 с. [Gelfand I. M., Shilov G.E. Generalized functions. Vol. 2: Spaces of fundamental and generalized functions. Moscow: Fizmatlit, 1958. 307 pp.]

5. Владимиров В.С., Волович Я.И. О нелинейном уравнении динамики в теории $p$ адической струны // TMФ, 2004. Т. 138, №3. С. 355-368; англ. пер.: Vladimirov V.S., Volovich Ya. I. Nonlinear Dynamics Equation in p-Adic String Theory // Theoret. and Math. Phys., 2004. Vol. 138, no. 3. Pp. 297-309, arXiv: math-ph/0306018.

6. Green M. B., Schwarz J., Witten E. Superstring theory. Vol. 1: Introduction / Cambridge Monographs on Mathematical Physics. Cambridge: Cambridge University Press, 1987. 469 рр.; русск. пер.: Грин М., Швари, Дж., Виттен Э. Теория суперструн. Т. I: Введение. М.: Мир, 1990. 518 c.; Green M. B., Schwarz J., Witten E. Superstring theory. Vol. 2: Loop amplitudes, anomalies and phenomenology / Cambridge Monographs on Mathematical Physics. Cambridge: Cambridge University Press, 1987. 596 pp.; русск. пер.: Грин М., Швари, Дж., Виттен Э. Теория суперструн. Т. ІІ: Петлевые амплитуды, аномалии и феноменология. М.: Мир, 1990. 656 с.

7. Brekke L., Freund P.G.O., Olson M., Witten E. Non-Archimedian string dynamics // Nuclear Phys. B, 1988. Vol. 302, no. 3. Pp. 365-402.

8. Frampton P. H., Okada Y. Effective scalar field theory of p-adic string // Phys. Rev. D (3), 1989. Vol. 37, no. 10. Pp. 3077-3079.

9. Владимиров В.С, Волович И. В., Зеленов Е.И. p-Адический анализ и математическая физика. М.: Физматлит, 1994. 352 с.; англ. пер.: Vladimirov V.S., Volovich I. V., Zelenov E. I. p-Adic analysis and mathematical physics. Vol.1/ Series on Soviet and East European Mathematics. Singapore: World Scientific Publishing Co., Inc., 1994. 319 pp.

10. Aref'eva I. Ja., Joukovskaja L. V., Koshelev A. S. Time evolution in superstring field theory on non-BPS brane. I. Rolling tachyon and energy-momentum conservation // J. High Energy Phys., 2003. no. 9, 012. 15 pp., arXiv: hep-th/0301137.

11. Aref'eva I. Ya. Nonlocal string tachyon as a model for cosmological dark energy/ In: AIP Conf. Proc., 826. Melville, NY: Amer. Inst. Phys., 2006. Pp. 301-311, arXiv: astro$\mathrm{ph} / 0410443$.

12. Calcagni G. Cosmological tachyon from cubic string field theory // J. High Energy Phys., 2006. no. 5, 012. 25 pp., arXiv: hep-th/0512259.

13. Владимиров В. С. О нелинейном уравнении $p$-адической открытой струны для скалярного поля // УMH, 2005. Т. 60, №6(366). С. 73-88; англ. пер.: Vladimirov V.S. On the non-linear equation of ap-adic open string for a scalar field // Russian Math. Surveys, 2005. Vol. 60, no. 6. Pp. 1077-1092. 
14. Moeller N., Zwiebach B. Dynamics with infinitely many time derivatives and rolling tachyons // J. High Energy Phys., 2002. no. 10, 034. 39 pp., arXiv: hep-th/0207107.

15. Sen A. Rolling Tachyon// J. High Energy Phys., 2002. no. 4, 048. 18 pp., arXiv: hepth/0203211.

16. Ghoshal D., Sen A. Tachyon condensation and brane descent relations in $p$-adic string theory // Nuclear Phys. B, 2000. Vol. 584, no. 1-2. Pp. 300-312.

17. Volovich I. V. p-Adic string// Class. Quantum Grav., 1987. Vol. 4, no.4. Pp. L83-L87.

18. Minahan J.A. Mode Interactions of the Tachyon Condensate in $p$-Adic String Theory // J. High Energy Phys., 2001. no. 3, 028. 16 pp.

19. Barnaby N. Caustic formation in tachyon effective field theories // J. High Energy Phys., 2004. no. 7, 025. 23 pp., arXiv: hep-th/0406120.

20. Coletti E., Sigalov I., Taylor W. Taming the Tachion in Cubic String Field Theory // J. High Energy Phys., 2005. no. 8, 104. 25 pp., arXiv: hep-th/0505031.

21. Владимиров B. C. Об уравнении $p$-адической открытой струны для скалярного поля тахионов // Изв. РАН. Сер. матем., 2005. Т. 69, № 3. С. 55-80; англ. пер.: Vladimirov V. S. The equation of the $p$-adic open string for the scalar tachyon field // Izv. Math., 2005. Vol. 69, no. 3. Pp. 487-512, arXiv: math-ph/0507018.

22. Vladimirov V.S. The equation of the $p$-adic closed strings for the scalar tachion field // Sci. China, Ser. A, 2008. Vol. 81, no. 4. Pp. 754-764.

23. Vladimirov V.S. On the Equations for $p$-Adic Closed and Open Strings // p-Adic Numbers, Ultrametric Analysis and Applications, 2009. Vol.1, no. 1. Pp. 79-87.

24. Владимиров В.С. О нелинейных уравнениях р-адических струн для скалярных полей тахионов/ В сб.: Избранные вопросы математической физики и р-адического анализа: Сборник статей/ Тр. МИАН, Т. 265. М.: МАИК, 2009. С. 254-272; англ. пер.: Vladimirov V.S. On nonlinear equations of $p$-adic strings for scalar tachyon fields // Proc. Steklov Inst. Math., 2009. Vol. 265. Pp. 242-261.

\section{MSC: 81T30}

\section{MATHEMATICAL QUESTIONS FOR THEORY OF NONLINEAR PSEUDODIFFERENTIAL EQUATIONS WITH p-ADIC STRING}

\section{V.S. Vladimirov}

Steklov Mathematical Institute, Russian Academy of Sciences, 8, Gubkina st., Moscow, 119991, Russia.

E-mail: vladim@mi.ras.ru

This work is devoted to the mathematical description of the dynamics of tachyons of open, closed and open-closed p-adic strings. The questions of existence and nonexistence of continuous solutions and their properties, as well zero structure of solutions is discussed. New multidimensional nonlinear equations of ultraparabolic type are obtained. Some unsolved problems are listed.

Key words: $p$-adic strings, nonlinear pseudodifferential equations, existence and continuity of solutions, zero structure of solutions.

Original article submitted $15 / \mathrm{XII} / 2010$ revision submitted 26/II/2011.

Vasiliy S. Vladimirov (Dr. Sci. (Phys. \& Math.), Academician), Chief Scientist, Consultant of RAS, Dept. of Mathematical Physics. 